\title{
ESTRÉS ACADÉMICO EN ESTUDIANTES UNIVERSITARIOS ASOCIADO A LA PANDEMIA POR COVID-19
}

ACADEMIC STRESS IN COLLEGE STUDENTES ASSOCIATED WITH COVID-19 PANDEMIC

Lilia González Velázquez

lilglez@gmail.com 
Para citar este artículo:

González Velázquez, L. (2020). Estrés académico en estudiantes universitarios asociados a la pandemia por COVID-19. ESPACIO I+D, INNOVACIÓN MÁS DESARROLLO, 9(25). https://doi. org/10.31644/IMASD.25.2020.a10

\section{RESUMEN}

El estrés académico asociado a la pandemia por la COVID-I9 se está incrementando. En el análisis preliminar de resultados de la Escala de Afrontamiento del Estrés Académico (A-CEA) y del Cuestionario Percepción del Estrés Académico en estudiantes universitarios asociado a la COVID-1 9 muestran una disminución de la motivación y del rendimiento académico con un aumento de la ansiedad y dificultades familiares, especialmente para estudiantes de escasos recursos económicos en una universidad pública del sur de México; se recupera el debate en la comunidad académica sobre el impacto que la epidemia está teniendo en la salud mental de los estudiantes y sus profesores, y cuáles son los retos que la educación superior tiene que enfrentar en un futuro inmediato.

\section{Palabras clave:}

Estrés académico, estudiantes universitarios, COVID- 19. 
-Abstract-

Academic stress associated with COVID-19 is increasing in college students. Preliminary data collected with the Escala de Afrontamiento del Estrés Académico (A-CEA) and Cuestionario Percepción del Estrés Académico en estudiantes universitarios asociado al COVID-19 shows a decrease in motivation and grades, and an increase in anxiety and family issues more acute for students with limited financial resources in a public university in southern Mexico. The article reviews current scholarship regarding the impact of epidemic on the mental health of students and faculty, as well as higher education challenges for the near future.

\section{Keywords:}

Academic stress, university students, COVID-19. 
ste artículo comparte la preocupación e interés por saber cuáles son los efectos emocionales identificados como estrés académico, debido al confinamiento y ruptura de la cotidianidad de la vida universitaria a raíz del cierre de la universidad y la obligatoriedad e realizar el proceso de enseñanza y aprendizaje en modalidad virtual. El documento está organizado en dos secciones, en la primera se hace una revisión de algunos de los planteamientos que expertos de diversas disciplinas están haciendo por medios virtuales, para advertir de los efectos negativos que ya se están detectando en la salud mental de niños y jóvenes, además de la preocupación por la pertinencia de las estrategias implementadas por las autoridades educativas para la continuidad académica, la afectación a la calidad en los procesos formativos, el abandono escolar y la sobrecarga de trabajo del profesorado.

Para brindar un ejemplo concreto de los efectos que la epidemia está provocando en los estudiantes, se muestran los resultados preliminares de una investigación, en curso, con la aplicación de dos instrumentos sobre estrés académico en estudiantes universitarios de la pedagogía de la UNACH que fueron aplicados en tres momentos: en septiembre de 2019, antes de la aparición de la pandemia, en abril de 2020 como fase inicial y de septiembre de 2020 llamada fase intermedia. ). La exposición de estos resultados es meramente ilustrativa y no es un reporte de investigación. Como un tema pendiente de investigar, se menciona el estrés en docentes - burnout. Finalmente, se hace una breve reflexión de las propuestas que se están vislumbrando para aprovechar los tiempos de crisis y reinventar la educación superior desde un enfoque innovador, humanista e integrador.

\section{EL ESCENARIO}

Desde el I I de marzo de 2020, fecha en que la OMS decretó oficialmente que el contagio por el virus COVID-19 sería una pandemia mundial como nunca vista antes, el mundo que conocíamos se detuvo súbitamente. Han pasado seis meses y las consecuencias del encierro forzado se pueden observar con mayor precisión. Hay consenso en señalar que son tres las áreas que más están siendo afectadas: en primer lugar la salud, ya se cuentan por miles las defunciones y por millones los contagios en todo el planeta; en segundo lugar está la economía, donde el daño no ha sido menor: pérdidas de millones de puestos de trabajo, cierre temporal o definitivo de empresas de todos los tamaños y giros, endeudamiento, etc. El tercer lugar lo tiene el sector educativo con la afectación de estudiantes de todos los niveles educativos.

En el ámbito de la educación, la pandemia en el mundo, ha afectado a más de 17 millones de estudiantes y a 80 millones de docentes, según informo Patricia Aldama; Directora de OEl-México, durante la inauguración el 03 de septiembre de este año, del Ciclo de Iberoamericano de Encuentros de 
Especialistas que analizan la situación de la pandemia en la educación (OEIIESALC, 2020). Por otro lado, la subsecretaría de educación superior de la SEP (2020) reporta que el país tiene una matrícula de cuatro millones y medio en licenciatura y 384,600 de posgrado y cerca de 400, 000 profesores, los cuales dejaron de tener actividades presenciales por la pandemia.

La repentina suspensión de clases presenciales tomó a todos desprevenidos: autoridades educativas, padres de familia, docentes y alumnos quienes, ante el inimaginable escenario y la repentina decisión de continuar a distancia los programas educativos, comprobaron que la magnitud de la tarea desafiaba los recursos disponibles, físicos, tecnológicos y humanos, generando ansiedad y estrés en un escenario de incertidumbre, miedo al contagio y preocupación por lo que vendrá en el futuro cercano.

En una publicación reciente de Dussel, Ferrante y Pulfer (2020), donde participan educadores latinoamericanos, presenta un mosaico de puntos de vista sobre los efectos de la pandemia en la educación en estudiantes, docentes, autoridades educativas, que reflejan la incertidumbre de este hecho jamás visto: millones de personas en aislamiento. Se percibe un consenso en los efectos negativos de la salud mental y las formas de interactuar en niños y jóvenes, por otro lado está el desgaste de los docentes y la oportunidad de reinventar la escuela y sus viejas inercias de lo que Freire llamó "educación bancaria".

En México, como en el mundo, los primeros estudios del efecto de la pandemia en la población aún se están escribiendo. Son muchas las preocupaciones de esta inédita situación, siendo la educación una de las más importantes. Las IES del país asumieron la gran responsabilidad de proteger la salud de sus comunidades académicas y anunciaron el cierre de todas sus instalaciones, sabedores de las afectaciones al ejercicio de sus funciones sustantivas (como la docencia, investigación y vinculación de servicios).

En la UNACH, suspendieron actividades presenciales el 22 de marzo de 2020 sus 22 mil 152 estudiantes de pregrado y 527 de posgrado, inscritos en alguno de los 79 programas de licenciatura, 16 de especialidad, 34 de maestría y II de doctorado que brindan formación profesional con 96 planes de estudio y 140 programas educativos. La oferta educativa atiende una matrícula total de 22 mil 679 estudiantes (UNACH, 20l8).

\section{BREVE CRONOLOGÍA DEL ESTRÉS ACADÉMICO EN UNA UNIVERSIDAD EN TIEMPOS DE LA COVID-19}

El estrés no es algo nuevo para los ciudadanos del siglo XXI, de hecho, desde el siglo pasado el crecimiento de las ciudades ha sido acelerado, en ellas viven más del $60 \%$ de la población mundial. Desde entonces, los ciudadanos han estado constantemente enfrentando numerosos cambios en su entorno, resultado del avance de la ciencia, la tecnología, la globalización 
de la economía, la estandarización de patrones culturales, el consumismo y el individualismo, así como por el dominio de un modelo educativo que se mantiene, en gran medida, presencial y tradicionalista. De cierta forma nos hemos acostumbrado a esa "normalidad": vivir con prisa, tener la constante presión por cumplir con las múltiples exigencias y actividades externas para mantener o mejorar nuestro nivel de vida y bienestar, poco tiempo queda para pensar en temas trascendentales, en nosotros mismos o en lo que pasa a los otros. La irrupción de la pandemia en el mundo, desestructuró abruptamente esa cotidianeidad. El confinamiento nos ha enfrentado a nosotros mismos, incrementando un extraño estrés a medida que la pandemia se fue prolongando.

Pero ¿qué es el estrés? Para los psicólogos es un síndrome. McKay, Davis y Fanning (1998: 9) lo definen como un "dolor emocional" producto de la "combinación de tres elementos: el ambiente, los pensamientos negativos y las respuestas físicas. Éstos interactúan de tal manera que hacen que una persona se sienta ansiosa, colérica o deprimida".

Lazarus y Folkman (1986) crearon un modelo cognitivo para evaluar el estrés, definiéndolo como un conjunto de relaciones que se establecen entre la valoración de una situación y la capacidad para enfrentarla. Esta relación genera tensiones y manifestaciones cognitivas, emocionales y conductuales que afectan la percepción del bienestar personal frente a un posible peligro, real o imaginado. El estrés tiene una función adaptativa que busca restablecer el equilibrio a nuevas circunstancias.

Barraza (2020: 19 y 20) comenta que las medidas de distanciamiento social, el confinamiento, el miedo al contagio y la suspensión de actividades sociales, laborales y recreativas, han generado niveles altos de estrés en la población mexicana; explica que este síndrome es un mecanismo adaptativo de los seres humanos, constituido por tres momentos: percepción, reacción y acción como respuesta:

a) el inicio de este proceso se da cuando el ser humano percibe las situaciones que representan un riesgo, una amenaza o un peligro para su integridad personal, b) ante esta situación potencialmente peligrosa, se presentan en el ser humano una serie de reacciones que sirven como alarma del inminente peligro que se cierne sobre él, y c) una vez identificada la situación potencialmente generadora de peligro y estando el cuerpo preparado para actuar sobreviene la acción para conservar su integridad personal.

Si bien el estrés puede ser positivo para mantener un equilibrio frente a los desafíos del entorno, cuando es intenso y prolongado en el tiempo puede tener consecuencias graves a largo plazo. La prolongación de la pandemia ha generado altos niveles de estrés, debido a la incertidumbre de cuándo y cómo terminará. Dado los recientes sucesos, aún no hay datos fehacientes 
sobre los efectos socioemocionales de la pandemia en la población y en particular en los estudiantes universitarios, por lo que a manera de ejemplo, recurro a narrar mi propia experiencia como docente y la de mis colegas cercanos en la Universidad Autónoma de Chiapas, una universidad pública del sur de México.

Para describir la situación de estrés en esta institución educativa, recurro a los tres momentos del estrés descritos por Barraza. El autor señala que el primer momento del estrés es la percepción del peligro. Durante los meses de febrero y marzo de 2020 la información de la pandemia se conoció por los noticieros. La información de los políticos y autoridades de la salud sobre el alcance de la pandemia y su duración, era contradictoria e incluso minimizada, a pesar de que otros países europeos y asiáticos reportaban numerosos contagios y las primeras muertes. La percepción del peligro fue aumentado hasta que a finales de marzo, la Secretaría de Educación Pública recomendó cerrar las escuelas de todos los niveles educativos. En el caso de las universidades casi todas atendieron el llamado y suspendieron clases presenciales a fines de marzo, dejando a consideración de cada facultad y carrera el decidir la forma de concluir el mes y medio faltante del semestre. Todavía en ese momento la percepción del peligro era dudosa, algunos pensaron que era una exageración y otros, que sería peor. En la UNACH, el cierre fue también repentino, los docentes improvisaron, de acuerdo a sus recursos y capacidades, cómo concluir el semestre. Algunos accedieron a plataformas como Classroom, otros videoconferencias por Zoom o Meet, otros simplemente dejaron tareas por correo electrónico y mensajes de What App a sus alumnos. En ese tiempo, muchos pensábamos que la pandemia no sería tan larga y se regresaría a clases presenciales para el siguiente semestre y todo quedaría en una anécdota histórica. Desafortunadamente no fue así, casi al inicio de las vacaciones de verano, las noticias del aumento vertiginoso de la pandemia en el mundo y en nuestro país, alertó a las autoridades académicas, quienes fueron aplazando una y otra vez, la fecha del inicio de las clases presenciales; la incertidumbre aumentó hasta que, finalmente, a fines de junio se anunció oficialmente que las clases y toda la actividad académica sería en línea el resto del año. El segundo momento del síndrome del estrés que señala Barraza, es la reacción ante la magnitud. La abrumadora cantidad de información de las autoridades de salud para seguir las reglas sanitarias, la magnificación del peligro del contagio por el virus que podía estar en cualquier lugar sin poder percibirlo, saber que no existe una vacuna, la sana distancia y el confinamiento, uso de mascarillas, etc. generó reacciones diversas: primero fue el miedo, enojo por el confinamiento, desconfianza, angustia, duelo por los seres queridos enfermos o que fallecieron por el contagio, etc. La poca mejoría del panorama, obligó a las autoridades a tomar la decisión de 
continuar el nuevo semestre de agosto-diciembre en la modalidad a distancia. Entonces, en junio y julio, de manera imperiosa, se comunicó a toda la planta docente de la institución, de licenciatura y posgrado, en todas las áreas del conocimiento: medicina, ciencias exactas, sociales, humanidades, ingenierías, arte y literatura, etc., que llevarían un curso en línea de una semana, para el manejo de la plataforma institucional Educa-T, que la Universidad utiliza para las carreras a distancia, pues era necesario adquirir las competencias digitales necesarias para, de inmediato, aplicarlas en el diseño instruccional de nuestras asignaturas. Yo participé en el primero de los 5 talleres, éramos 432 docentes con competencias digitales muy dispares: la mayoría no conocía ningún tipo de plataformas, ni mucho menos había hecho diseño instruccional para alguna de sus asignaturas, ni usado recursos tecnológicos y didácticos para educación a distancia, tampoco usado videoconferencias para las clases. En ese momento, la planta docente dimensionó el enorme reto que tenía enfrente, lo que le generó angustia, ansiedad y resistencia.

Podemos hablar de una fase intermedia de la pandemia, que ha sido ubicada en el segundo trimestre del año. En el caso de la Universidad, inició a fines de junio, julio, y continúa con el regreso a la clases virtuales a fines de agosto. Se puede decir que la percepción de la gravedad de la situación en la comunidad universitaria fue cambiando con un aumento significativo del estrés por los meses de confinamiento, el distanciamiento social, el arduo trabajo de los docentes para "subir a la plataforma" sus asignaturas durante las "vacaciones de verano", y la obligación de continuar las clases en la modalidad presencial. La persistencia de la situación sanitaria y las alteraciones de las rutinas de la vida universitaria, ha tenido efectos más negativos que positivos, pues tanto colegas, como estudiantes y personal administrativo expresaban sus dudas e incertidumbre por las consecuencias de estas medidas. Muchos piensan que es un semestre perdido, que el aprovechamiento de los estudiantes ha bajado y poco se podrá recuperar, por lo que prefieren la modalidad presencial.

Para todos, sin duda, la pandemia de la COVID ha sido difícil, no solo por el riesgo del contagio, sino por la incertidumbre de no saber cuándo terminará y si se podrá vivir de nuevo sin miedo. En un libro reciente sobre la situación de la educación en tiempos de la pandemia, hecho a gran velocidad por docentes iberoamericanos, se resalta que el cierre de las escuelas es un acontecimiento traumático para los actores educativos; no se sabe a ciencia cierta cuáles serán los efectos, si al regresar se reinstalarán las viejas prácticas y se volverá a la "vieja normalidad" o la "nueva normalidad" será beneficiosa para innovar y romper inercias, y si el sistema se reinventará o, como lo que mencionan, permanecerá la "escuela profunda" caracterizada por la desigualdad (Dussel, Ferrante y Pulfer, 2020). 
El análisis del complejo panorama se hace con el fin de hacer un recuento de los daños, construir nuevas interpretaciones y vislumbrar propuestas de solución, eso es lo que está en marcha. En los últimos tres meses se han organizado rápidamente numerosos foros y publicaciones en línea, disponibles para todos. Entre las principales preocupaciones, los especialistas mencionan el aumento de las diferencias socioeconómicas que puedan poner en riesgo de abandono escolar a los estudiantes en situación económica desventajosa, ya que, como siempre sucede en nuestro país, en momentos de crisis los sectores de la población más pobres son los más afectados.A ello hay que agregar el ahondamiento de la brecha tecnológica, por la falta de dinero de las familias para comprar una computadora, tableta 0 simplemente tener una televisión para ver los programas de la SEP; además de no contar con internet o por vivir en zonas geográficas de difícil acceso (Foro virtual: Tensiones entre educación y educación inclusiva. Desafíos a partir de la COVID, 2020; ISSUE; 2020; COMIE a y b).

En el reciente Informe del Instituto Internacional de la UNESCO para la Educación Superior en América Latina y el Caribe (IESALC, 2020: 16), se menciona cuáles pueden ser los impactos de la pandemia previsibles a corto, medio y largo plazo para los estudiantes de educación superior en el tema de la salud emocional:

Inevitablemente, la pérdida de contacto social y de las rutinas de socialización que forman parte de la experiencia cotidiana de un estudiante de educación superior tendrán un costo. El aislamiento que va inevitablemente asociado al confinamiento tendrá efectos en términos de equilibrio socioemocional que dejarán huella, en particular, en aquellos estudiantes con problemáticas preexistentes en este dominio. A los estudiantes más vulnerables que participan en programas de nivelación y apoyo, el aislamiento les golpea aún más fuerte. Indicativamente, una encuesta realizada la última semana de marzo entre estudiantes de educación superior en Estados Unidos3 ha revelado que un 75\% afirma haber experimentado ansiedad y depresión como resultado de la crisis (p. 16).

Otros de los aspectos que se están presentado, son los efectos emocionales en los sujetos, primero el confinamiento prolongado donde se interrumpen la interacción social esencial para las personas, el distanciamiento de los compañeros de clase donde se construyen relaciones amistosas, amigos y el ambiente de la vida universitaria es preocupante. También hay que agregar que en la vida familiar, las situaciones que se viven son diversas y también fuente de estrés, pues hay padres que han perdido su empleo, lo que genera violencia intrafamiliar, la convivencia puede ser difícil ya que los otros espacios se han limitado. 


\section{ESTRÉS ACADÉMICO EN LA UNIVERSIDAD. LOS ESTUDIANTES}

El estrés académico se ha definido como la situación de tensión física y emocional vinculada a las exigencias del mundo académico. La mayoría de las investigaciones abordan el estrés de los estudiantes y poco el de los docentes. Respecto a los primeros se han estudiando sus efectos en el rendimiento académico (Fernández de Calvo y Luévano, 2018; Feldman, et al, 2008), en el autoconcepto académico (Martín, 2007; Muñoz, 1999), en las creencias de autoeficacia y la salud (Pereyra, Paez, Del Valle, Agustina, 2019; Pulido et al, 20II) y en general, las afectaciones que provoca en la salud física y mental de los estudiantes y docentes.

La percepción del estudiante sobre su capacidad para enfrentar exitosamente las exigencias académicas, pueden generar pensamientos y emociones negativas, afectación física como sueño, dolor de cabeza, ansiedad, desconcentración, desorganización y problemas de adaptación. Se encuentra que hay una relación proporcional entre el autoconcepto académico y la susceptibilidad al estrés. Fernández de Castro y Luévano (2018), en una reciente investigación con estudiantes mexicanos, encontraron que la fuente más fuerte de estrés académico en los estudiantes está relacionado con las calificaciones, más que con la obtención del conocimiento, por eso es en el periodo de exámenes cuando se registran los índices más altos de inestabilidad emocional y repentinas enfermedades. En menor grado, se encuentran como estresores la habilidad para solucionar problemas, la tolerancia a la frustración o a las exigencias de tiempo y esfuerzo de los docentes y de las asignaturas.

Sin duda, a las conocidas fuentes de estrés académico, los nuevos acontecimientos han agregado otras, propias de las situaciones de guerra o peligro grave, como el miedo irracional al contagio, el distanciamiento social que lleva al aislamiento y la desconfianza, la incertidumbre y disminución del esfuerzo, constancia, alegría y seguridad. Tal vez por eso también el regreso al seno de la familia tiene varias lecturas en los jóvenes, por un lado, la percepción de recibir alto apoyo social pero, por otra, pérdida de independencia y de los espacios propios de la interacción universitaria con los amigos, compañeros de clase y docentes.

El repentino cambio a la modalidad de educación a distancia, provocó un cierto desamparo que afecta los anteriores niveles de motivación. Otros estudios recientes, en países como los árabes y asiáticos, han identificado como factores protectores la religiosidad, la familia y el apoyo de los profesores (Pajarianto, et al, 2020).

En septiembre se marcó el regreso a clases, pero a distancia, y así será hasta concluir en diciembre. La situación de los estudiantes es diversa, por ejemplo, los chicos que ingresan por primera vez a la universidad 
solo conocen a sus profesores y compañeros a través de la pantalla de la computadora o de los celulares, esto chicos son población especialmente vulnerable a este situación, son los estudiantes de los primeros semestres los que están más expuestos al estrés académico, por estar en un proceso de integración (Pulido, et al, 20II).

Los que ya habían cursado más semestres en modalidad presencial, saben que no podrán convivir con sus compañeros varios meses más. Se agrega el temor de no contar con una buena computadora y recursos para pagar el internet y mantenerse conectados para recibir las clases, tema muy presente en las escuelas públicas y en los estudiantes que están en comunidades de difícil acceso, poder reprobar o tener una baja calificación por este hecho es una fuente de estrés. Al respecto, López y Rodríguez (2020: 103) señalan que existe el riesgo de que estudiantes mexicanos puedan ser afectados de manera importante en su trayectoria académica e incluso provocar su deserción:

A esto se suma que, aunque existe la creencia de que estos alumnos pertenecen a la generación tecnológica, muchos de ellos carecen de las habilidades necesarias para desarrollar actividades de aprendizaje de forma virtual, así como de la autodisciplina que demandan estas modalidades, en un ambiente de estrés, incertidumbre y de restricciones socioeconómicas derivadas de la pandemia.

\section{AVANCES DE INVESTIGACIÓN A MANERA DE EJEMPLO. ESTRÉS ACADÉMICO DE LOS ESTUDIANTES DE LA UNACH EN EL ESCENARIO LA PANDEMIA}

Con el interés de medir el estrés académico de los estudiantes, en septiembre del año pasado inicié un proyecto de investigación que se ha ampliado a partir de la aparición de la contingencia sanitaria. Con este fin, se seleccionó el instrumento Escala de Afrontamiento del Estrés Académico (A-CEA) de Cabanach, et al (20I0) que explora en qué grado el estudiante usa ciertas estrategias de afrontamiento cognitivas, conductuales y sociales frente a situaciones de estrés académico; consta de 23 preguntas que se responden con una escala tipo Likert que va de Nunca a Siempre. De acuerdo a los autores, se miden dos estrategias de afrontamiento básicas:

(a) las estrategias orientadas al problema o activas, representadas en las dimensiones de reevaluación positiva y planificación, estrategias que posibilitan la solución del problema y la consiguiente eliminación del estrés; (b) el afrontamiento orientado a la emoción, medido por la dimensión de búsqueda de ayuda, y especialmente del apoyo social y emocional, cuyo efecto de amortiguación del estrés es ampliamente aceptado por la comunidad científica (p. 59). 
En septiembre de 2019, la Escala se aplicó a un grupo de 40 estudiantes de la carrera de pedagogía de la Universidad Autónoma de Chiapas (UNACH), México. Los resultados indicaron bajo estrés académico, que se incrementaba en temporada de exámenes o en la exposición de temas frente a sus compañeros; la utilización de estrategias de afrontamiento activo se reporta en un $60 \%$ de los estudiantes, lo que indica que cuentan con habilidades para autorregular su comportamiento y se sienten capaces de controlar los factores que les pueden generar estrés, mientras que el resto hace más uso de estrategias de apoyo social y emocional social, que indican cierta dependencia en los demás y escasas habilidades de autorregulación para enfrentar situaciones estresantes. (González y González, 2019).

Con la aparición de la contingencia sanitaria y el cierre de la Universidad, se consideró oportuno explorar si había cambios en las estrategias de afrontamiento de estrés académico, además de identificar en los estudiantes aspectos estresantes asociados con la situación del confinamiento y el cambio de la manera de estudiar, ahora en la modalidad en línea. Dado que la Escala no contempla el tema de la COVID-19, se diseñó el Cuestionario Percepción del Estrés Académico en Estudiantes Universitarios asociado a la COVID-I 9 (PEA_COVID-19) (González, L. y Pech, S., 2020).

Ambos instrumentos fueron aplicados en línea a 72 estudiantes en abril de 2020 y a 94 en septiembre del mismo año, en la reanudación del nuevo semestre. Es pertinente señalar que si bien los participantes en las tres aplicaciones no son las mismas personas, comparten muchas similitudes como son la edad ( 19 a 21 años), proporción hombre y mujeres (77\% de mujeres y $31 \%$ hombres, promedio), nivel socioeconómico (medio-bajo), carrera (Pedagogía) y Universidad (UNACH). Los resultados de la comparación de los tres momentos (pre contingencia, inicial y actual) están en proceso, pero se pueden mencionar algunos preliminares, para ejemplificar si hay un cambio en la percepción de los jóvenes de la situación traumática que les tocó vivir en los últimos meses y que seguramente continuará, por lo menos, hasta diciembre de este año.

A manera de ejemplo, ya que este artículo no es un informe de investigación, se seleccionaron algunos ítems representativos del tema que nos interesa. En las Figuras I y 2 se muestran los tres momentos de aplicación de Escala de Estrategias de Afrontamiento de Estrés Académico (A-CEA) con las respuestas a dos preguntas clave que reflejan si hay percepción de factores estresores. 
6. Cuando me enfrento a una situación problemática mientras estoy preparando los exámenes o una tarea complicada, planifico detalladamente cómo estudiar el examen o hacer el trabajo

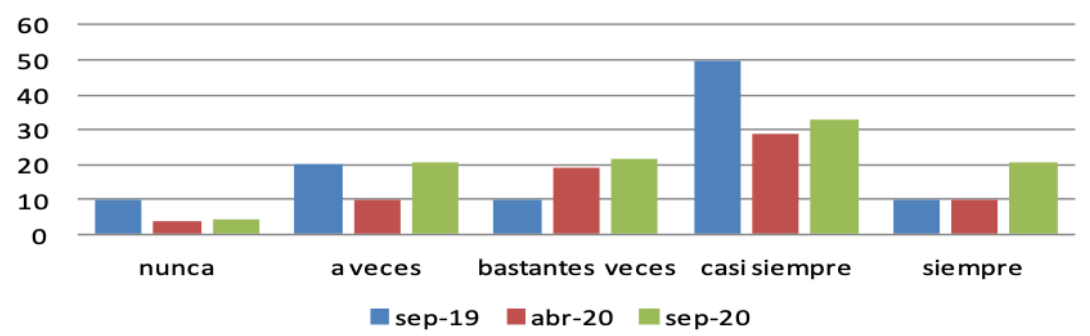

Figura 1. Porcentaje de respuesta a la pregunta 6 de la Escala A-CEA. Fuente: elaboración propia

Como se ha dicho antes, una de las situaciones que más causa estrés en los estudiantes es la presentación de un examen. Como se observa en la Figura I, en la aplicación en 2019 , más de la mitad de los chicos reportaron contar con suficientes estrategias para mantener una actitud positiva y de control frente a una situación complicada, como un examen, preparando con anticipación su preparación, lo que puede implicar un estrés bajo. Si comparamos con los estudiantes, ya en tiempos de la pandemia, hay diversidad en las respuestas lo que denota una posible desorganización en el estudio, al haberse alterado las rutinas y espacios académicos, la percepción de pérdida de control, puede incrementar en estrés académico.
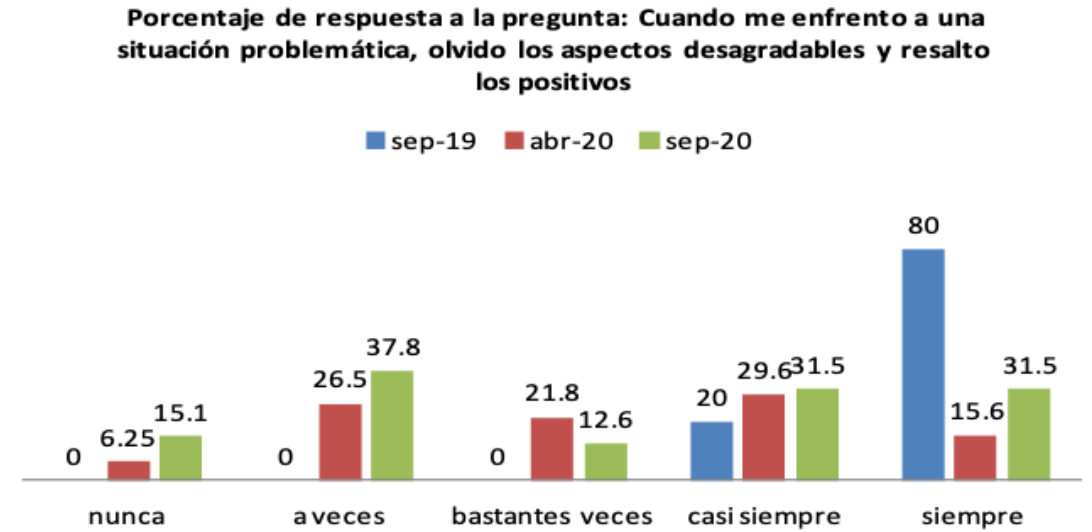

Figura 2. Porcentaje de respuesta en la pregunta 1 de la Escala A-CEA. Fuente: elaboración propia

La capacidad de diferir los pensamientos negativos y mantener un enfoque positivo frente a los problemas, implica utilizar estrategias de afrontamiento activo. En la Figura 2 se puede observar, en los tres momentos, cambios significativos. Antes de la pandemia, los estudiantes dicen mantener un enfoque positivo frente a los problemas, lo que contrasta con los dos grupos de participantes en tiempos del confinamiento y las clases a distancia donde 
un poco más de la tercera parte tiene emociones y pensamientos negativos, lo que es preocupante. En un estudio posterior, González et al (2018:429-430) autores del A-CEA confirman nuevamente que:

los estudiantes que hacen un menor uso de las estrategias de afrontamiento activo son los más vulnerables al estrés académico y los que manifiestan un mayor malestar. Es posible que ello tenga alguna relación con el hecho de que el estudiante percibe muchas de las situaciones académicas como controlables al menos, las más significativas en su desempeño académico, es decir, situaciones en las que sabemos de forma consciente que algo podemos hacer, considerando, por tanto, que puede enfrentarse a ellas, empleando mecanismos de autorregulación que actúen positivamente sobre sus efectos psicofisiológicos y le permitan mantener su equilibrio y su adaptación.

Con el propósito de tener información específica de los estudiantes de la Universidad respecto a cómo estaban viviendo la situación de la continencia sanitaria, se elaboró el Cuestionario Percepción del Estrés Académico en Estudiantes Universitarios asociado a la COVID- 19 (González, y Pech, 2020) integrado por 20 ítems que recuperan datos socio demográficos familiares, recursos tecnológicos para los cursos en línea, niveles de estrés académico percibido, desempeño de los docentes, salud física, entre otros.

Este instrumento se aplicó en línea en abril de 2020 a un mes y medio del cierre del semestre, con actividades no presenciales, y en septiembre del mismo año, con el inicio de clases en la plataforma EDUCA-T de la Universidad. Como se ha comentado, esta investigación está en curso, por lo que nuevamente se presentarán algunos datos con el fin de contextualizar en un caso las consecuencias de esta inédita situación y cómo afecta emocionalmente a nuestros estudiantes. Los datos socio demográficos de los estudiantes en la Universidad, en un estado de la Republica Mexicana como es Chiapas, considerado como uno de los de mayor rezago educativo, pobreza y dispersión geográfica de su población. De acuerdo a los datos proporcionados, se tiene que la escolaridad de los padres es baja, en el caso del padre el 2,2 \% no tiene ningún estudio, $20.2 \%$ tiene primaria, el 34.8 $\%$ secundaria, el $26 \%$ bachillerato y el resto licenciatura, en el caso de las madres, estos porcentajes son menores en todos los casos; la ocupación de los padres es en el campo, empleados poco calificados, pequeños comerciantes, lo que significa vivir con muchas restricciones económicas. Esto explica el hecho de que el $35.8 \%$ de los estudiantes participantes no cuenten con una computadora personal, que el $35 \%$ no tenga acceso a internet o sea muy irregular el servicio. A la pregunta de la afectación que tuvieron por el cambio a las clases en línea, es decir si había afectado su rendimiento académico, el $16.8 \%$ dijo que mucho y el $58.4 \%$ poco. Un porcentaje muy parecido reportaron al responder por la afectación a su motivación para 
estudiar. La evaluación del desempeño de sus docentes, el $28 \%$ lo calificó de deficiente o muy deficiente, mientras que el resto se mostró satisfecho con su actuación. En cuanto a la experiencia de trabajar en la plataforma, solo el $12.4 \%$ manifestaron que se adaptaron rápido y le gustó la modalidad, el resto expresaron su malestar en diversos grados y esperar regresar a las clases presenciales. De manera reiterada, los jóvenes expresaron su preocupación por el riesgo de bajar en su promedio, al no poder estar en todas las clases virtuales, no poder entregar sus trabajos a tiempo y seguir teniendo dificultades para moverse con facilidad en la plataforma. En las preguntas abiertas, comentan que extrañan a sus amigos, estar en el ambiente universitario y poder consultar con sus profesores y que le puedan responder sus dudas de manera inmediata.

Finalmente, en esta selección de preguntas, se les pidió que hicieran una valoración general su percepción del estrés académico. En la Figura 3 se muestra las respuestas en el grupo de estudiantes del mes de abril, que se corresponde con la fase inicial de la pandemia y en la Figura 4, las respuestas del grupo de septiembre, considerada como la fase intermedia de este fenómeno.

Durante la crisis y el confinamiento, me he sentido, (Escala de uno (1) _ muy mal a diez (10) _muy bien...

72 respuestas

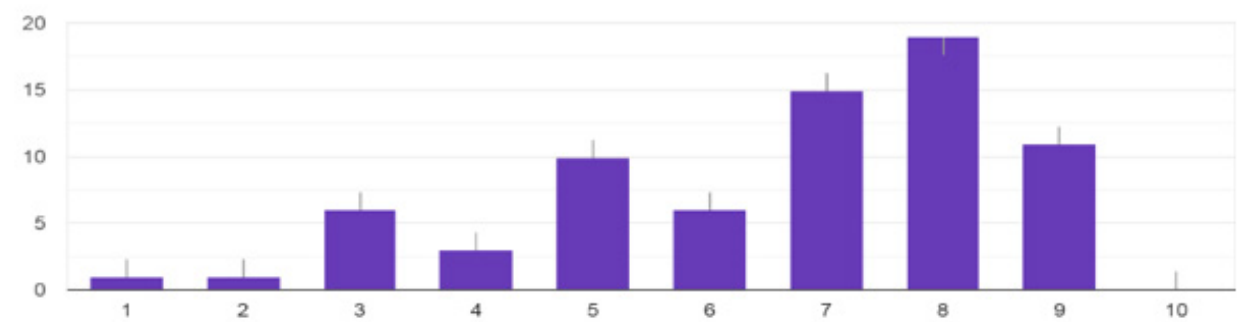

Figura 3. Frecuencias de respuesta del grupo abril-20 al PEA_COVID-19. Fuente: elaboración propia

Durante la crisis y el confinamiento, me he sentido en una escala del 1 al 10,donde 1 es muy mal y 10 muy bien (sep 2020)

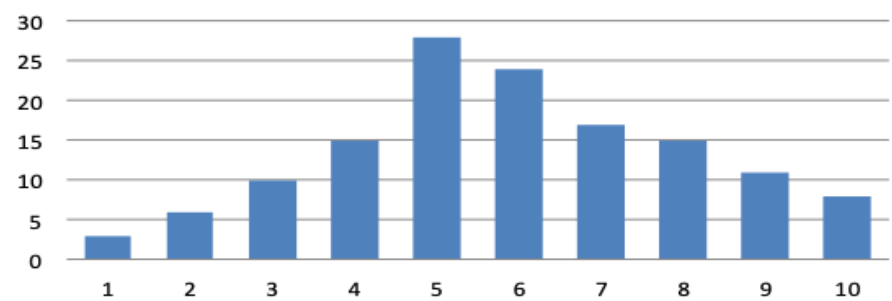

Figura 4. Frecuencias de respuesta del grupo septiembre-20 al PEA_COVID-19. Fuente: elaboración propia 
En la comparación de ambas figuras nos podemos dar cuenta que, si bien los estudiantes son capaces de manejar el estrés académico, sí está registrándose un incremento importante, lo que en algunos casos puede afectar la trayectoria escolar de estos jóvenes.

\section{LOS DOCENTES}

En el informe preparado por el Instituto Internacional de la UNESCO para la Educación Superior en América Latina y el Caribe (UNESCO - IESALC, 2020:26), en el marco de la pandemia por la COVID-19, dice lo siguiente: "Aunque el foco se coloca siempre en los impactos sobre los estudiantes, el profesorado sufre también importantes afectaciones en lo laboral y en lo profesional (...) el impacto más evidente sobre los docentes está siendo la expectativa, cuando no exigencia, de la continuidad de la actividad docente bajo la modalidad virtual".

El estrés de docente se ha relacionado con el malestar docente o el síndrome de burnout. Desde la década de los setenta se han realizado numerosas investigaciones que dan cuenta del desgaste físico y emocional de los profesores. Se reconoce que la docencia es una de las profesiones más estresantes y a la vez, más incomprendidas y reconocidas.

Al respecto Zavala (2008:7I) señala que:

La manifestación del burnout en grupos de riesgo como lo son los docentes aparece cuando el estrés es crónico y los mecanismos de afrontamiento del individuo no son los más adecuados. El burnout también puede interpretarse como una capacidad de respuesta no adecuada ante una diversidad de demandas no satisfactorias que pudieran ser desde un trabajo monótono y aburrido, hasta una labor cuyos constantes requerimientos de conocimiento sean excesivos demasiados conflictos emocionales, situaciones de no valoración laboral y personal e inclusive pobre remuneración.

La relación del estrés con el síndrome de burnout o del "quemado o quemarse" es estrecha. Existe suficiente evidencia de que el desgaste laboral afecta la salud física y emocional de las personas. Cabrera y Colina (2019), hacen una revisión del estado del arte de las investigaciones sobre el estrés del profesor en la región de Latinoamérica, con el consenso de que "las exigencias institucionales, como elevar la calidad de la enseñanza y la sobrecarga de trabajo, son condiciones estresantes y vehículos para el desarrollo de estrés crónico. El estrés convertido en síndrome de quemarse en el trabajo tiene impacto en el desempeño docente" (p.l0).

El estrés del profesor no es exclusivo de algún nivel educativo, aunque sí es más intenso en educación básica, donde los grupos numerosos y la presión de las autoridades por cumplir con el programa es constante. En 
educación superior, el profesor tiene más flexibilidad con la "libertad de cátedra" y tal vez menos alumnos, sin embargo, desde hace dos décadas, las políticas de certificación de los programas educativos y la carrera docente, han sometido a los profesores a la frenética carrera de la productividad académica y así incrementar los bajos salarios que reciben.

Por su naturaleza, la profesión docente es de larga trayectoria y con relativa poca movilidad una vez que el docente ha logrado definitividad en su plaza. En estos casos, la mayoría ejerce más de 30 años en una misma institución, espacio donde se consolidan grandes amistades, pero también discordias políticas, ideológicas y académicas entre los colegas. Al paso del tiempo, va apareciendo el estrés crónico o burnout.

No es sorpresivo que el estrés docente haya incrementado significativamente a partir de la aparición de la pandemia a principios de este año. En este artículo se comparten algunas situaciones que se vivieron en la Universidad, debido a esta contingencia sanitaria, y también datos preliminares de la identificación del estrés académico en los estudiantes. Aunque no hay aún una medición, es muy posible que los docentes también estén sufriendo de mayores niveles de ansiedad, como sus alumnos, al ver alterado abruptamente su concepción epistemológica y didáctica del enseñar y aprender; lo que es entendible si consideramos que por siglos, el modelo de docencia dominante se ha caracterizado por mucho tiempo por ser presencial, centrada en el profesor, ejercida en espacios físicos (aulas, laboratorios, talleres, etc. ) con evaluaciones que verifican mayormente la retención de información y contenidos curriculares enciclopédicos, no siempre actualizados, que deben ser "aprendidos" por los estudiantes de la misma manera y al mismo ritmo y tiempo. En el Informe de UNESCOIESALC (2020:26) en el que se analiza la compleja y difícil situación que enfrentan muchos docentes ante la urgencia de trabajar con tecnologías que antes no consideraban necesarias ni eran de su interés, da lugar incluso a nuevas terminologías:

Coronateaching que se ha definido como el proceso de "transformar las clases presenciales a modo virtual, pero sin cambiar el currículum ni la metodología". Esta entrada abrupta en una modalidad docente compleja, con múltiples opciones tecnológicas y pedagógicas, y con una curva de aprendizaje pronunciada puede saldarse con resultados poco óptimos, frustración y agobio debido a la adaptación a una modalidad educativa nunca antes experimentada sin la correspondiente capacitación para ello. Pero el término Coronoteaching también se utiliza para referirse a un fenómeno socioeducativo emergente con implicaciones psico-afectivas, tanto en profesores como en estudiantes.

ESPACIO I+D, INNOVACIÓN MÁS DESARROLLO • Vol. IX, No. 25, Especial COVID-19, 2020 • ISSN: 2007-6703 


\section{EL FUTURO DE LA EDUCACIÓN SUPERIOR: ¿RENOVARSE O MORIR?}

Hemos visto que el intempestivo cambio de modalidad educativa, ha expuesto a estudiantes y docentes a altos niveles de estrés y malestar, y nadie sabe aún cuáles serán las consecuencias cuando se abra de nuevo la universidad.

Parece ser que hay una fuerte tendencia en la comunidad académica mundial que apuesta por hacer una lectura adecuada de las lecciones implícitas que la pandemia nos ha mostrado y aprovechar la oportunidad de renovar las prácticas educativas y fortalecer a sus actores. En una conferencia reciente, Ángel Díaz Barriga (2020) va por este enfoque y oportunidad de cambio; afirma que en adelante, las IES serán b learning y que el aprendizaje forzado de las tecnologías que tuvieron que hacer estudiantes y docentes para la continuidad académica, se quedarán y fortalecerán, coadyuvando a que los estudiantes sean personas autónomas en su aprendizaje y los profesores hagan una "docencia distribuida" que promueva el pensamiento divergente y contenidos curriculares clave. En la misma dirección López y Rodríguez (2020:108) consideran que "Esta situación tendría que considerarse como una oportunidad que contribuya a la reflexión sobre la flexibilidad de las prácticas del proceso de enseñanza-aprendizaje, para que los estudiantes desarrollen capacidades de autoaprendizaje, los docentes seamos una guía en este proceso y no solo transmisores sistemáticos de contenidos, y las IES fomenten el desarrollo de estas prácticas educativas".

Es claro que la formación que reciben nuestros universitarios está lejos de ser "integral" como la mayoría de los modelos educativos institucionales pregonan pero que, en la práctica, no impulsan programas para fortalecer sus habilidades cognitivas y socioafectivas, lo que les deja en un estado permanente de vulnerabilidad frente a las presiones y problemas de la vida $y$ de su desempeño y permanencia, cuando se debería fortalecer su capacidad de resiliencia académica (Gómez y Rivas 2017).

Lozano, et al (2020:96) en su reciente investigación sobre los impactos del COVIS-19 comentan que "la conclusión más destacada de este estudio exploratorio consiste en constatar el fuerte impacto que el confinamiento ha tenido para la situación vital del estudiantado universitario en el ámbito psicológico y académico, principalmente, y la relación existente con la satisfacción vital y la resiliencia, fundamentalmente".

$Y$ es que una de las lecciones que nos está dejando esta pandemia es en la aldea global, nadie está asegurado de escapar a hechos catastróficos de cualquier índole, sea ambiental, económico, político, sanitario, social y educativo, por mencionar algunos. Ha quedado demostrado que la educación ha formado a los estudiantes y los docentes no tienen las competencias necesarias para enfrentar lo inédito, complejo y peligroso. En este sentido, la inclusión 
en el currículo universitario de espacios formativos para el desarrollo de habilidades de pensamiento crítico, resolución de problemas, autorregulación del aprendizaje, auto eficacia, inteligencia emocional y resiliencia académica, es un asunto que no debe seguir siendo minimizado. Por ello y para estar en una mejor posición para enfrentar nuevas catástrofes que sin duda vendrán, las instituciones de educación superior están obligada a replantear a fondo su modelo educativo y formar otro tipo de ciudadanos y profesionales que sepan reaccionar de mejor manera no solo en situaciones de emergencia sino en la construcción de un mundo mejor.

\section{REFLEXIONES FINALES}

Dicen que los tiempos de crisis son momentos en que se puede alcanzar o retroceder, pero nunca se vuelve al mismo sitio. De la misma manera. La magnitud de la pandemia de la COVID- 19 cimbró al mundo y aún no termina. Las pérdidas para la economía y el mercado del trabajo se estiman enormes y tardarán años en recuperarse. En educación, la afectación es también de grandes dimensiones, aunque más difícil de cuantificar. Igual como sucede en un terremoto, que echa abajo edificios con cimientos débiles y viejos, la pandemia nos sacudió y dejó al descubierto lo que ya sabíamos: el modelo de educación es obsoleto, vulnerable y excluyente. Este panorama abre enormes e interesantes líneas de investigación educativa, que deben atender los educadores, autoridades educativas y políticos, sin dilación (COMIE, 2020).

Es urgente no apuntalar el edificio educativo, sino reconstruirlo con otro modelo pertinente a los nuevos tiempos, complejos, cambiantes e inciertos, pero iserá posible, o una vez pasado el susto regresaremos a nuestros viejos hábitos, a nuestras zonas de confort por malas que estas sean? Estas y otras interrogantes se están estado debatiendo en el mundo de la educación y, sí, también genera estrés académico. 


\section{REFERENCIAS}

Barraza, A. (junio, 2020). El estrés de pandemia (COVID 19) en la población mexicana. Centro de Estudios Clínica e Investigación Psicoanalítica S.C, México. Disponible en http://www.upd.edu.mx/PDF/Libros/Coronavirus.pdf

Cabanach, Ramón G.; Valle, Antonio; Rodríguez, Susana; Piñeiro, Isabel; Freire, Carlos (2010). Escala de afrontamiento del estrés académico (A-CEA). Revista Iberoamericana de Psicología y Salud, vol. 1, núm. 1, enero, pp. 5164. Disponible en: http://www.redalyc.org/articulo.oa?id=245116411005

Cabrera, H., y Colina, A. (2019). Estrés en profesores universitarios. Debates en evaluación y currículum. Congreso Internacional de Educación: Curriculum 2019/Año 5, No. 5, septiembre 2019 a Agosto de 2020.

COMIE-a (2020). Foro virtual de análisis: La investigación educativa en tiempos del COVID-19 24 de junio. Disponible en: https://www.youtube.com/ watch?v=kmDxp4-Ye-U

COMIE-b (2020) Tensiones entre educación y educación inclusiva. Desafíos a partir del COVID-19, 25 de junio. Disponible en: https://www.youtube. $\mathrm{com} /$ watch?v=p4aTtP36WSo

Díaz Barriga, Ángel (2020). Conferencias educativas Educación A Distancia. Nuevos Retos En Los Modelos Educativos. Educación superior. 14 jun. 2020. Disponible en: https://www.youtube.com/ watch?v=ZBdvwa3Gt5w

Dussel, I., Ferrante, P. y Pulfer, D. (2020). Pensar la educación en tiempos de pandemia. UNIPE, Editorial Universitaria. Disponible en: http://redesib. formacionib.org/grupos/docentes-frente-a-la-pandemia/blog/pensa

Feldman, L.,Goncalves, L., Chacón-Puignau, C., Zaragoza,j., Bagés, N., y De Pablo, J. (2008). Relaciones entre estrés académico, apoyo social, salud mental y rendimiento académico en estudiantes universitarios venezolanos. Universitas Psychologica v. 7 no. 3, septiembre-diciembre.

Fernández de Castro Javier y Luévano, Edith (2018). Influencia del Estrés Académico sobre el Rendimiento Escolar en Educación Media Superior. Revista Panamericana de Pedagogía, Núm. 26, 97-117.

Gómez, G y Rivas, M. (2017). Resiliencia académica, nuevas perspectivas de interpretación del aprendizaje en contextos de vulnerabilidad social. Calidad en la Educación. No 47, pp. 215-233.

González Cabanach, R.; Souto-Gestal, A.; González-Doniz, L. y Franco Taboada, V. (2018). Perfiles de afrontamiento y estrés académico en estudiantes universitarios. Revista de Investigación Educativa, 3 (2), 421-433. DOI: http://dx.doi.org/10.6018/rie.36.2.290901

González, L. y González, M.R. (2019). Salud y estrés académico en estudiantes universitarios. Ponencia presentada en el IX Congreso Internacional 
Internacional Salud, Bienestar y Sociedad. Berkeley, San Francisco, California, USA, 19 y 20 de septiembre.

González, L. y Pech, S. J. (2020). Cuestionario Percepción del Estrés Académico en Estudiantes Universitarios asociado a la COVID-19. (Documento interno no publicado).

ISSUE (2020). Educación y Pandemia. Una Visión Académica. UNAM, México. Disponible en: http://www.iisue.unam.iisue./covid/educacion-y-pandemia consultado 25 de mayo de 2020.

ISSUE-UNAM (2020). Educación y pandemia: el futuro que vendrá/Hugo Casanova. Director ejecutivo•Emitido en directo el 18 jun. 2020. Disponible en: https://www.youtube.com/watch?v=yt_78IYBKf4.

Ibermón, F. (2020). Conferencia magistral: formación continua y desarrollo profesional docente, presentado en el Ciclo Iberoamericano de Encuentros con Especialistas. OEI-MEJOREDU_SEP. Disponible en: http://youtube.be/4WNs5MnCH6E

Lazarus, R. S. y Folkman, S. (1986). Estrés y procesos cognitivos. Barcelona: Martínez-Roca.

López, M., y Rodríguez, S. A. (2020). Trayectorias escolares en la educación superior ante la pandemia ¿continuar, interrumpir o desistir? En H. Casanova Cardiel (Coord.), Educación y pandemia: una visión académica (pp. 103108). UNAM, Ciudad de México, Instituto de Investigaciones sobre la Universidad y la Educación. http://www.iisue.unam.iisue./covid/educaciony-pandemia consultado 25 de mayo de 2020.

Lozano Díaz, A., Fernández-Prados, J.S., Figueredo Canosa, V. \& Martínez Martínez, A.M. (2020 Impactos del confinamiento por el COVID-19 entre universitarios: Satisfacción Vital, Resiliencia y Capital Social Online. International Journal of Sociology of Education, Special Issue: COVID-19 Crisis and Socioeducative Inequalities and Strategies to Overcome them, 79-104. http://doi.org/10.17583/rise.2020.5925

Martin, M. (2007). Estrés académico en estudiantes universitarios. Apuntes de Psicología, Vol. 25, número 1, Universidad de Sevilla.

McKay, M, Davis, M., y Fanning, O. (1998). Técnicas cognitivas para el tratamiento del estrés. Barcelona: Martínez Roca.

Pajarianto1, E., Kadir, A, Galugu, N.,Sari, P.,\& Februanti, S. (2020). Study from Home in the Middle of the COVID-19 Pandemic: Analysis of Religiosity, Teacher, and Parents Support Against Academic Stress. Talent Development \& Excellence Vol.12, No.2s, 2020, 1791-1807

OEI-IESALC (2020, mayo 13). COVID-19 y Educación Superior: De los efectos inmediatos al día después. Análisis de impactos, respuestas políticas y recomendaciones. Disponible en: http://www.iesalc.unesco.org/wpcontent/uploads/2020/05/COVID-19-ES-130520.pdf 
Ordorika, I. (2020). Pandemia y Educación Superior. Revista de la Educación Superior 194, Vol. 49, pp. 1-8, Disponible en: https://doi.org/10.36857/ resu.2020.194.1120

SEP (2020) Portal de la Subsecretaría de Educación Superior, consulta el 10 de septiembre. Disponible en: https://educacionsuperior.sep.gob.mx/

UNACH (2018). Proyecto Académico, Reforma para la Excelencia 2018-2012. UNACH, Tuxtla Gutiérrez, Chiapas, México. Disponible en: http://www. unach.mx

Zavala, J. (2008). Estrés y burnout docente: conceptos, causas y efectos. Educación Vol. XVII, No. 32, marzo 2008, pp. 67-86. Disponible en: http://revistas.pucp.edu.pe/index.php/educacion/article/view/1802 